\title{
ONICOFAGIA Y COVID-19: ESTUDIO DE CASO DE UN JOVEN ADULTO CON TDHA
}

\author{
J. Inmaculada Sánchez Casado \\ Universidad de Extremadura \\ iscasado@unex.es \\ Elena I. Benítez Sánchez \\ Universidad de Extremadura \\ ebenitezx@alumnos.unex.es
}

Recepción Artículo: 16 mayo 2021 Admisión Evaluación: 16 mayo 2021 Informe Evaluador 1: 12 mayo 2021 Informe Evaluador 2: 23 mayo 2021 Aprobación Publicación: 01 junio 2021

\section{RESUMEN}

La onicofagia es una conducta poco adaptativa que presentan algunos sujetos; esta patología les provoca una necesidad de "morder" compulsivamente sus uñas. Si no se trata precozmente tiende a perpetuarse en el tiempo, ya que es una tendencia que no disminuye con la edad, y se ve incrementada significativamente ante situaciones de estrés o de ansiedad. En este estudio de caso se analizará la intervención cognitivo-conductual con un adulto de 25 años diagnosticado de TDAH, cuyo objetivo responde a la necesidad de erradicar la conducta de morderse las uñas durante esta actual etapa pandémica, con la finalidad última de garantizar las recomendaciones más básicas del protocolo COVID-19.

Este tipo de conductas, muy peligrosas por la transmisión del virus a través de las manos, son especialmente frecuentes en los pacientes con trastornos del desarrollo, y especialmente en jóvenes con TDAH, como en nuestro caso.

La intervención, empleando como terapia el método cognitivo-conductual con reforzadores negativos y positivos e infografías de la Consejería de Sanidad de la Junta de Extremadura, que recalcaban la importancia de las medidas básicas de salud e higiene de manos, ha alcanzado una alta tasa de éxito. Este hecho viene avalado por la eficiencia y eficacia de la propia terapia, que duró 90 días, y que se implementó durante el periodo de confinamiento domiciliario provocado por la pandemia Covid-19 entre los meses de marzo, abril, mayo y junio. Los resultados mostraron una disminución paulatina de la frecuencia de morderse las uñas, siendo exponencial desde la primera semana hasta erradicar la misma en las últimas semanas de tratamiento.

Palabras clave: Onicofagía; joven TDHA;intervención; covid-19 


\section{ABSTRACT}

Onychophagia and COVID-19: Case study of a young adult with ADHD. The "nail bitting" is a behaviour, which is not very adaptive, and it is presented by some subjects. This pathology provoques on them the necessity of bitting their nails compulsively. If it is not treated precociously tends to perpetuate itself in time, and it is a tendency which is not decreasing with the age. It can be also increased in situations of stress and anxiety. This study will analyze the cognitive-conductual intervention with an adult of 25 years old, who is diagnosed of A.D.D.. The main objective of this research responds to the necessity of eradicate the behaviour of biting his nails during this pandemic stage. The end point of this investigation is to guarantee the fulfillment of the most basic recommendations on the COVID-19 protocol.

This type of conduct are very dangerous because of the way the virus is mainly transmitted, which is through the hands, the nail bitting is overly frequent in patients with development issues, particularly those who are afflicted of A.D.D. like ours subject.

The intervention who has used cognitive-conductual therapy with negative and positive reinforcers besides infographies of "Consejería de Sanidad de la Junta de Extremadura" emphasising the importance of the basics health measures and hand hygiene has had a high rate of success. The efficacy and efficiency of the therapy is endorsed by itself and it has evolved in a 90 days' period and which was implemented during the home comfinement provoqued by the COVID-19 pandemic in March, April, May and June. The results demonstrated a slowly decrease of the frequency of nail bitting, acquiring an exponential rate since the first week until the complete eradication in the last weeks of treatment.

Keywords: "Nail bitting"; adult of 25 years old A.D.D.; intervention; covid-19

\section{INTRODUCCIÓN}

La onicofagia es el hábito de morderse las uñas, normalmente, las uñas de las manos, sin preferencia por ningún dedo en concreto. Suele ser algo temporal en los niños y su condición como conducta patológica va a depender de la frecuencia, la intensidad y la duración de la misma. (Ghanizadeh, 2011) Este comportamiento crónico afecta al 20-30\% de la población general, sin embargo parece ser un problema completamente ignorado en la práctica clínica diaria. (Psacan et al., 2014) Quizás porque en la sociedad actual no suele tener una repercusión importante para el desempeño profesional en la edad adulta.

Las conductas repetitivas y centradas u orientadas en el cuerpo aluden a todos aquellos comportamientos que se centran en la remoción intencional de partes del organismo, tales como uñas, piel, cabello y mucosas; se presentan de manera frecuente en la infancia y la adolescencia.

La mayoría no son patológicas y se consideran como un "hábito", una peculiar forma de estar, de manifestarse al exterior, de conseguir un psedoequilibrio cinestésico básico, es decir, como una conducta reiterada, egosintónica y con un propósito determinado que está bajo el control de la voluntad.

Sin embargo, cuando la conducta se torna persistente, ciertamente viciosa y la persona es incapaz de evitarla pese a la voluntad de hacerlo, con el consecuente y significativo deterioro funcional y social, se vuelve "patológica". Tal conducta recobra vida propia, no suple una determinada necesidad, y por ende se convierte en el centro vital del individuo que no es capaz de dominar, se vuelve compulsiva y generadora de sentimientos de culpa, de desconcierto, y de falta de control sobre su propia vida. La onicofagia puede considerarse como una conducta poco adaptativa por el impacto que supone en la vida de los adultos jóvenes, aunque no sólo en el colectivo en el que se centra este estudio, sino por las graves repercusiones en el devenir de los individuos (Pelios et al., 1999), sobre todo por generar como indicaron Cortese y Biondi, en el 2009, (Cortese \& Biondi, 2009) hábitos parafuncionales, en el sentido de centrarse con rigor en el propio cuerpo de forma repetitiva. Es un hábito recurrente patológico, sin finalidad placentera, al contrario, podemos conceptualizarlo com una forma de estar en el mundo disfuncional.

La onicofagia presenta también gradientes, es decir, puede ser más intensa y compleja, en el sentido de que puede extenderse "el hecho de morder" a una, varias o a todas las uñas de los dedos tanto de una sola mano 
como de las dos manos del paciente, y es común que exista asimismo el mordisqueo de las cutículas, lo que provoca la formación de los padrastros. Este hábito es socialmente inaceptable ya que produce desagrado en quienes entran en contacto con la persona que se muerde las uñas; desde la propia visión pública del acto, un poco desagradable que además, tiene implicaciones que van más allá de su falta de atractivo, pues produce problemas médicos y dentales principalmente cuando se sobrepasa el borde libre de la uña. (Ghanizadeh, 2011)

Algunas de las consecuencias más destacadas podríamos enumerarlas siguiendo a Lorena del Encinar Cano Lucas (2018) como :

- Paroniquia y lesiones de la matriz ungueal que pueden conducir a la pérdida total de la uña.

- Alteraciones dentarias (resorción del esmalte dental, maloclusión) y lesiones en las encías.

- Síndrome temporomandibular.

- Infecciones por enterobacterias y otros gérmenes presentes en las uñas, por el transporte de estos al tubo digestivo. Y una de las posibles consecuencias más sobredimensionadas en el periodo actual de pandemia por la COVID-19 que va enmarcar todo nuestro estudio.

Para (García, 2006) las causas más frecuentes que llevan a la persona al hábito de morderse las uñas son los estados obsesivo-compulsivos, el estrés y la agresividad; son, así, formas mediante las cuales las personas se calman en momentos de tensión, ansiedad y angustia, o cuando se encuentran en un estado de desadaptación provocado por cambios en la unidad familiar o la pérdida de seres queridos, entre otras. Cualquiera de estos estados puede desencadenar automáticamente y de manera frenética un impulso de morderse las uñas. Y de ahí que este problema en los tiempos actuales no sea una cuestión baladí, sino por el contrario, un tema que merece toda nuestra atención porque puede desencadenar que la persona pueda contraer el virus, por no ser capaz de gestionar esta simple medida de seguridad y evitar así la transmisión del mismo por contacto manual.

En cuanto a la posible incidencia de la onicofagia por sexo, hemos de manifestar que no existen diferencias de género en niños menores de 10 años, pero en la adolescencia, es más frecuente que se dé en varones, probablemente, porque las mujeres se preocupan más por la estética, por su imagen, y está claro que esta conducta perniciosa no es admitida socialmente y ese factor puede ayudarlas a tratarlo, y a aplicar mecanismos de contención y compensación. (Lorena del Encinar Cano Lucas, 2018)

En los tiempos actuales que corren debido a la Pandemia de Covid-19, la importancia de la higiene de manos, ha aumentado considerablemente, principalmente debido a que uno de los medios de transmisión es el contacto directo con superficie y con nuestra cara. El que la persona vea la necesidad de dejar de morderse las uños, es algo que en ocasiones no se alcanza ni siquiera durante la madurez del afectado, sin embargo esta nueva situación puede ser considerada como un potente detonante, gracias a las numerosas infografias empleadas por el SES, recalcando la importancia de no tocarse la cara, y realizar cuidadosamente la higiene de manos. 
Figura 1: Infografía del SES: Importancia de NO tocarse la cara y realizar una adecuada higiene de manos. (Infografías Del SES Higiene Manos - Búsqueda de Google, n.d.)

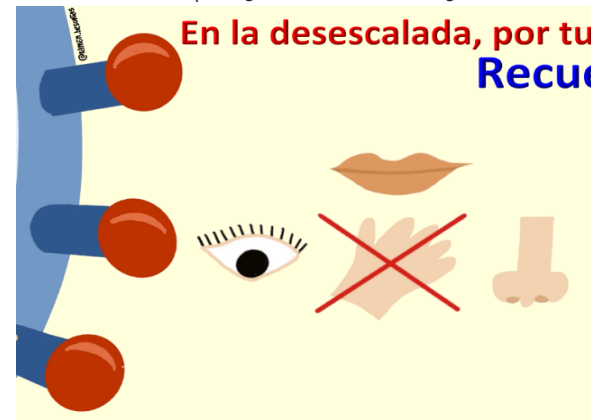

No toques la cara

- Si las manos están contaminadas, podrías inocularte el virus.

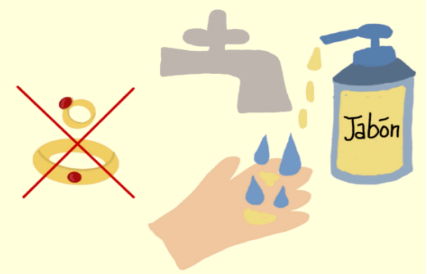

Lávate las manos

- Usa agua y jabón o un desinfectante a base de alcohol.

- No te confíes si llevas guantes.

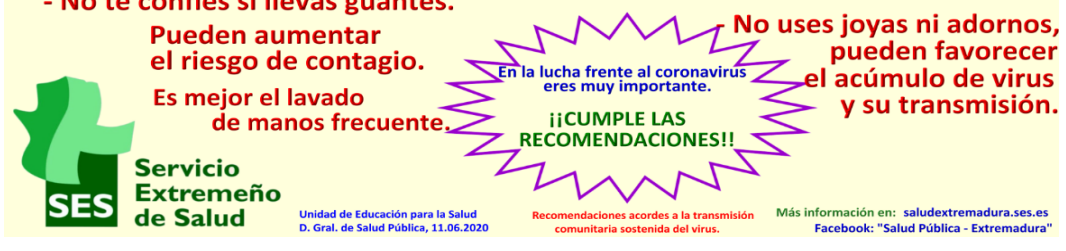

MÉTODO

\section{Sujeto}

Un varón de 25 años, alumno de la Universidad de Extremadura, estudiante del doble Grado de Educación Infantil y Primaria. Residente en la ciudad de Mérida, que presentaba esta conducta desde que recuerda hasta la fecha de la investigación en la que se produjo la intervención. Mostraba callosidades en el costado de los dedos, inflamación en la zona ungueal y padrastros recurrentes en los bordes laterales de la uña. El joven había sido diagnosticado de TDAH en su adolescencia, y presentaba un buen manejo del mismo, sin que éste condicionara en exceso su rendimiento académico. Aunque sí, que le provocaba mayor sensación de estrés y ansiedad, ante situaciones académicas demandantes más frecuentemente que el resto de sus compañeros.

\section{Instrumento}

Para valorar inicialmente a nuestro paciente, a fin de registrar diariamente la frecuencia de morderse las uñas se ha diseñado un autorregistro específico, (en Psicología México Cortés Aguado \& Tena, 2011) en el que el propio paciente enumeraba las veces que conscientemente se detectaba teniendo el comportamiento, también indicada la actividad que se encontraba realizando, el estado emocional y si creía que había un desencadenante de esa emoción. Tales parámetros fueron fáciles de interiorizar por el paciente y su aplicación fue del mismo modo muy efectiva.

Así mismo se han empleado entrevistas donde se ha hablado con el paciente, valorando qué tipo de situaciones él identificaba o creía que podían ser desencadenantes de sus conductas onicofágicas. También se habló de cómo manejaba la frustración y la ira o el posible estrés, poniendo un especial énfasis en la enumeración de sus estrategias de afrontamiento para las mismas, ya que la actitud en las sesiones de intervención fue muy colaboradora y estaba muy sensibilizado de erradicar estas conductas por el contexto pandémico en que nos encontrábamos. A esta situación ad hoc se le suma toda la exposición de redes sociales, medios de comunicación y la 
campaña liderada por el Servicio Extremeño de Salud (SES) y apoyado su difusión por las diferentes Facultades de la Universidad de Extremadura en referencia a la importancia de lavarse las manos, no tocarse la cara y los riesgos que subrayan que podría tener un hábito como éste, específicamente de morderse las uñas, en tiempos de pandemia por COVID-19.

\section{Procedimiento}

Se le solicitó un consentimiento informado para participar en la investigación y una vez que aceptó, comenzó la primera fase de la intervención donde se le proporcionó el autorregistro que el paciente completó durante los primeros 15 días. Así mismo, se reforzó su objetivo de erradicar este hábito perjudicial con las infografías del $S E S$, que recalcaban la importancia de no tener contacto entre las manos y las zonas con mucosas, que aumentaban drásticamente el riesgo de contagio. A continuación se acordaron con el paciente una serie de elementos de ayuda así como reforzadores positivos y negativos tanto sociales (apreciación de la mejora, refuerzo de la apariencia positiva de las manos ante la ausencia de conductas onicofágicas... ) como materiales (elegir las tareas domésticas a realizar, elegir el almuerzo o la cena en los casos positivos o sacar la basura, fregar los platos en los casos negativos) para llevar a cabo en la etapa de la intervención. (Salgado Serrano \& Oropeza, 2012)

A partir del día 16 se inició la segunda fase de la investigación, la intervención directa, donde se aplicó el tratamiento conductual. En primer lugar se procedió a pintar las uñas de las dos manos con una sustancia ("MORDEX", esmalte amargo y transparente dispensado en una farmacia, cuya característica principal es su fuerte sabor amargo) durante 2 veces al día, además de aplicarse los reforzadores positivos de tipo social como por ejemplo la posibilidad de elegir la temática de la película que se vería tras la cena en el caso de que las conductas medias onicofágicas estuvieran por debajo del objetivo fijado esa semana. En el caso de que el número de conductas estuviera por encima del objetivo se le aplicaba un refuerzo negativo (recoger la cocina, fregar los platos, sacar la basura,...).

Durante toda la intervención se observó que los refuerzos positivos eran más eficaces en cuanto a la extinción de la conducta que los negativos, ya que normalmente se producía una reducción de la conducta. Y esa tendencia la consideramos muy positiva, por lo que conllevaba para el tratamiento pero también por la garantía de eliminar riesgos de contagios del virus. Los reforzadores negativos se aplicaron un total de 3 veces. Así mismo, el esmalte de uñas con sabor amargo se continuó utilizando durante las 10 semanas de intervención directa. La justificación que permitió esta decisión técnica quedaba ampliamente avalada por el peligro que este tipo de conducta que se pretendía erradicar. El efecto del contagio es una posibilidad exponencial importante para este tipo de pacientes onicofágicos.

A partir de la semana 11 se comenzó con la fase de seguimiento que tuvo una duración de 15 días consecutivos a fin de seguir monitoreando la conducta onicofágica y corroborar el mantenimiento de los resultados obtenidos. Durante esta fase, el paciente continuó haciendo el registro diario de la conducta y siempre que ésta no se produjera se mantenían los reforzadores positivos para garantizar la tasa de éxito del 100\% como era necesario de instaurar.

\section{RESULTADOS}

\section{Estado base}

Para valorar las características conductuales del paciente se tuvo en cuenta durante las 2 primeras semanas cómo, cuándo, y con qué intensidad se producían las conductas onicofágicas. Se observó una frecuencia promedio de las conductas onicofágicas conscientes por parte del sujeto que rondaban la treintena. Estas conductas se producían en momentos que el sujeto identificaba como de estrés, cuando se trataba algún tema importante que le resultaba molesto, ante situaciones donde tenía que dar una respuesta rápida 0 ante momentos de exigencias académicas (en clase telemática que era el sistema impuesto durante el confinamiento, realizando un trabajo en grupo,...) 
Gráfico 1: Evolución de las conductas onicofágicas a lo largo de intervención cognitivo-conductual.

Gráfica de Dispersión 1: Relación temporal entre las semanas de intervención y el $n^{0}$ de conductas onicofágicas medias presentadas.

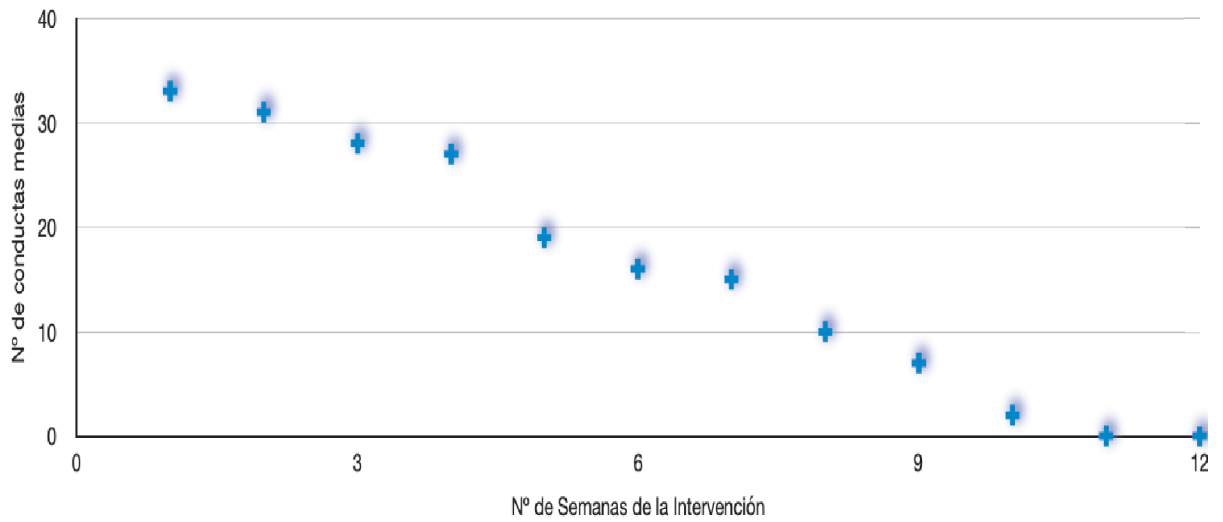

Tabla 1: № de conductas onicofágicas en función de las semanas de intervención.

\begin{tabular}{|c|c}
\hline $\boldsymbol{N}^{\boldsymbol{o}}$ de Semanas de intervención & $\boldsymbol{N}^{\boldsymbol{o}}$ de conductas onicofágicas \\
\hline 1 & 33 \\
\hline 2 & 31 \\
\hline 3 & 28 \\
\hline 4 & 27 \\
\hline 5 & 19 \\
\hline 6 & 16 \\
\hline 7 & 15 \\
\hline 8 & 10 \\
\hline 9 & 7 \\
\hline 10 & 2 \\
\hline 11 & 0 \\
\hline 12 & 0 \\
\hline
\end{tabular}

\section{Tratamiento}

A partir del día 15 se comenzó el tratamiento donde se aplicaron todos los reforzadores y que tuvo una duración de 9 semanas consecutivas. Es importante reseñar que se aprovechó todo el periodo de confinamiento, con lo que esta variable ha sido tremendamente positiva para activar el cambio de mirada del propio paciente hacia el peligro y la necesidad de erradicar ese comportamiento usual. 
El sujeto respondió muy favorablemente a la intervención, presentaba una actitud muy receptiva en las sesiones celebradas a través de la plataforma zoom, y observándose así un descenso notable ante la suma de los reforzadores y la intervención directa con el esmalte de uñas amargo (MORDEX) dos veces al día durante toda la exposición al tratamiento.

Las conductas onicofágicas se redujeron mejorando el estado de las uñas y reduciendo las inflamaciones periungueales, prácticamente desde la cuarta semana.

En la semana 10 de intervención las conductas onicofágicas estaban casi extintas, no refiriendo el paciente necesidad de morderse las uñas ante situaciones estresantes. Y esto es digno de destacar porque el final del curso pandémico en confinamiento ha puesto a prueba la salud mental de toda la población estudiantil, ya que la mayoría de los universitarios tuvieron que realizar un cambio vertiginoso desde la presencialidad más tradicional a un modelo on-line, donde los propios exámenes fueron realizados así, no solo la impartición de clases del segundo semestre. Esta situación desencadenó un impacto, considerado negativo; una "prueba de fuego", por así decirlo, para el éxito del tratamiento aplicado en este joven con TDAH, que por sus necesidades de apoyo educativo específico plantea un nivel de exigencia aún mayor para regularizar esta conducta inapropiada como era morderse las uñas desde las primeras etapas de su vida, algo que reconocía en la anamnesis realizada.

\section{Seguimiento}

El seguimiento se realizó durante 15 días posteriores a la extinción casi completa de las conductas durante las cuales se continuó el autorregistro para corroborar la verdadera eliminación del hábito. La terapia cognitivoconductual aplicada desde marzo a junio pretendía que la tasa de éxito completa fuera garantizada como necesidad per se de esta investigación, ya que era la única fórmula de que el paciente con TDAH permaneciese a salvo de los riesgos inherentes a los contagios por manipulación de superficies contaminadas. Si ya no se mordía las uñas en ninguna situación y bajo ningún concepto, el objetivo final cumplía los niveles de salud total que se perseguían con el tratamiento.

\section{Entrevista final}

Como último punto señalar que se ha realizado al final del procedimiento una entrevista acerca de la intervención aplicada; en ella se pudo comprobar cómo el paciente se mostraba aliviado por haber logrado dejar de morderse las uñas, ya que este hábito recurrente condicionaba la percepción que los demás podían tener sobre su carácter, así como las posibles repercusiones profesionales futuras. Al estudiar algunas asignaturas relacionadas con la psicología en el Grado de Maestro, pudo comprobar cómo estas manifestaciones que él era incapaz de erradicar le iban a condicionar sus futuras prácticas externas, al mismo tiempo que esa toma de conciencia le hacían sentirse "culpable" por no poder dejar de realizarlas en público, bien en clase 0 incluso en algunos momentos más de asueto con la familia, amigos,... Además por supuesto, de la eliminación de todas las complicaciones médicas que presentaba en las uñas debido a este hábito, que eran obvias simplemente al escribir en el ordenador, dibujar, tocar la guitarra, tocarse la mascarilla,...; principalmente se habían reducido las molestias que presentaba en los dedos que limitaban alguna de sus actividades diarias, sobre todo debido a las infecciones recurrentes, inflamaciones periungueales y los padrastros.

\section{DISCUSIÓN}

Esta investigación se ha realizado para eliminar el hábito onicofágico en un joven adulto de 25 años. Los episodios de onicofagia tenían una duración muy baja, pero eran muy numerosos durante el día especialmente. La frecuencia de los mismos comenzaron a disminuir a partir de la segunda semana hasta conseguir eliminar la conducta que se había mantenido desde su niñez de acuerdo a la anamnesis realizada al comienzo de la intervención por medio de la plataforma zoom.

Con el registro diario el paciente pudo darse cuenta qué situaciones desencadenaron el hábito (conflicto familiar, estrés ante determinadas tareas domésticas, o universitarias, ansiedad anticipatoria cuando tenía que 


\section{ONICOFAGIA Y COVID-19: ESTUDIO DE CASO DE UN JOVEN ADULTO CON TDHA}

planificar un trabajo con otros compañeros, ...) así como los pensamientos o emociones que le acompañaban en esos momentos (miedo, ira, frustración, desánimo, angustia,... ) además de registrar la frecuencia media en la que se producía la conducta.

Las diversas estrategias conductuales empleadas fueron muy efectivas, pero lo que resultó determinante como reforzador negativo fue la aplicación del esmalte de uñas con sabor amargo ya que evitaba que se reproduciese el patrón recurrente de nuestro paciente, donde se generaban episodios de poca intensidad pero muy repetidos coincidiendo casi siempre con situaciones de mayor estrés y ansiedad sobre todo académicas o por conflictos familiares. Cosa que era muy común en el tiempo de confinamiento en que tuvo lugar la intervención. También se apreció que los reforzadores negativos como tener que fregar la loza de la cena o sacar la basura eran menos efectivos que los reforzadores positivos, ya que cuando éstos se producían se incrementaba las respuestas favorables en el paciente.

Esta terapia de intervención se ha basado en otras realizadas por diversos autores como Lorena del Encinar Cano Lucas (2018); Salgado Serrano \& Oropeza, 2012); Cortés Aguado \& Tena (2011), la cual ha sido utilizado con éxito en diversos tratamientos conductuales, como la tricotilomanía (Moreno Vázquez et al., 2007) además de otros pacientes con enfermedades tipo hipertensión o ansiedad, que han reforzado el autocontrol de los usuarios favoreciendo así la autonomía de la persona frente a sus hábitos negativos. Este tipo de terapia de intervención se ha probado en numerosos estudios como más efectiva que las modificaciones realizadas a largo plazo sobre todo en niños y adolescentes.

\section{CONCLUSIÓN}

El análisis del estudio de caso que nos ocupa se ha vertebrado en torno a la onicofagia que un paciente joven adulto con TDAH padecía. La aplicación de una terapia cognitivo-conductual, con reforzadores y empleo de intervenciones directas de tipo cuasi-farmacológico (esmalte de uñas con sabor amargo) se ha llevado a cabo durante 90 días, en los meses de marzo, abril, mayo y junio de 2020.

Lo interesante de esta investigación es que ha tenido lugar durante el periodo pandémico, un contexto modulador que ha condicionado toda la estrategia de trabajo, ya que la manipulación de diferentes superficies y el hecho de que el paciente se mordiera las uñas sin control, provocaba un riesgo importante para contraer la infección por la COVID-19. Campañas lideradas desde el Servicio Extremeño de Salud y divulgadas por la propia institución universitaria, la UEx, impulsaron que el sujeto estuviera dispuesto a participar en un tratamiento aprovechando el propio confinamiento a través de sesiones directas por zoom con el terapeuta.

La onicofagia es un hábito perjudicial que puede condicionar muchas situaciones sociales del día a día, además resulta especialmente frecuente en pacientes con ciertos trastorno del neurodesarrollo, especialmente en el TDAH que es una condición intrínseca de este joven estudiante universitario.

En esa dirección se ha comprobado que este tipo de hábito suele estar relacionado con los eventos en el que el paciente siente ansiedad, estrés, frustración, presión,... sobre todo en el caso relacionado con las demandas académicas del segundo semestre del Grado de Maestro. Una circunstancia muy especial que cumple una doble condición. La primera que genera en el usuario una mayor carga de trabajo, un manejo de la incertidumbre útil y unas exigencias telemáticas nuevas pero no ajustadas a la realidad tradicional universitaria presencial. Por otro, esa mezcla entre ansiedad extraña y algo de miedo que dimana del periodo de confinamiento ante el riesgo que supone contraer la enfermedad de la COVID-19 si no te tiene en cuenta una rigurosa higiene de manos, empuja la desazón del propio estudiante para sentirse más impelido a poner freno a este hábito desadaptativo recurrente de morderse la uñas, cuya aparición era bastante alejado en su historia de vida y que por ello lo tenía muy instaurado en su curso vital cotidiano, a pesar de padecer problemas periungueales y padrastros de forma visible y desagradable.

El tratamiento cognitivo-conductual aplicado durante los 90 días que duró el confinamiento más riguroso se ha mostrado altamente efectivo, ya que se ha comprobado una reducción media de las conductas durante las primeras semanas para acabar extinguiéndose al final del proceso. La extinción se ha mantenido durante el segui- 
miento, lo que demuestra la efectividad de la terapia. Y como recalcan algunos autores el entrenamiento para la reversión de la conducta ha sido más efectivo que el entrenamiento de manipulación objetivo, además ha permitido una consolidación a largo plazo mucho mayor con respecto a este último. (Ghanizadeh et al., 2013)

La onicofagia es, por tanto, una de las patologías más comunes, y su erradicación no es siempre sencilla, ya que requiere voluntariedad por parte del sujeto y una serie de condiciones básicas, de ahí la importancia de desarrollar terapias nuevas e investigar en intervenciones que se demuestran efectivas en un periodo de tiempo relativamente breve para facilitar su mantenimiento y adherencia especialmente en personas adultas con TDAH y niños 0 adolescentes.

La utilización de las redes sociales, la sensibilización de los medios de comunicación, y las infografías del SES en relación a la higiene de manos han modificado sensiblemente la necesidad de erradicar esta conducta perniciosa ya que el confinamiento, por un lado disparaba el riesgo de contagio, pero por otro también ha visibilizado la necesidad de erradicar dicha patología en el menor tiempo posible para poder asegurar y garantizar que el usuario no contrajera la enfermedad del COVID-19 en uno de los episodios automáticos de morderse las uñas tras haber manipulado cualquier objeto de uso cotidiano. Era, pues una necesidad vital, que en estos tiempos de pandemia es preciso reconocer. Antes era una conducta desadaptativa y perniciosa, ahora sin embargo se ha convertido en una conducta peligrosa y que puede desencadenar una enfermedad grave, por ser este tipo de pacientes, más vulnerables en el entorno actual pandémico.

\section{REFERENCIAS BIBLIOGRÁFICAS}

Cortese, S. G., \& Biondi, A. M. (2009). Relationship between dysfunctions and parafunctional oral habits, and temporomandibular disorders in children and teenagers. Archivos Argentinos de Pediatría, 107(2), 134-138. https://doi.org/10.1590/S0325-00752009000200007

Cortés Aguado, I. A., \& Tena, O. (2011). Enseñanza e Investigación en Psicología Consejo Nacional para la Enseñanza en. E Investigación En Psicología, 16(1), 103-113. http://www.redalyc.org/articulo.oa?id=29215963009

García, J. L. (2006). Orientaciones para la Onicofagia. Consejería de Educación, Cultura y Deporte.

Ghanizadeh, A. (2011). Nail biting; etiology, consequences and management. In Iranian Journal of Medical Sciences (Vol. 36, Issue 2, pp. 73-79). Shriaz University of Medical Sciences. /pmc/articles/PMC3556753/

Ghanizadeh, A., Bazrafshan, A., Firoozabadi, A., \& Dehbozorgi, G. (2013). Habit reversal versus object manipulation training for treating nail biting: A randomized controlled clinical trial. Iranian Journal of Psychiatry, 8(2), 61-67. https://pubmed.ncbi.nlm.nih.gov/24130603/

Infografías del SES higiene manos - Búsqueda de Google. (n.d.). Retrieved May 5, 2021, from https://www.google.com/search?q=infografías+del+SES+higiene+manos\&tbm=isch\&ved=2ahUKEwjmkJrVwbLwAhUBhRoK $\begin{array}{lllllllll}H & Y & Y & S & C & 5 & Y & Q & 2\end{array}$ cCegQIABAA\&oq=infografías+del+SES+higiene+manos\&gs_Icp=CgNpbWcQA1Dxb1jnhgFgg4gBaABwAHg AgAFZiAGQCZIBAjEOmAEAoAEBqgELZ3dzLXdpei1pbWfAAQE\&sclient=img\&ei=mYuSYKbgPIGKaobZrLAJ $\&$ bih $=892 \&$ biw $=1920 \#$ imgrc $=0$ CYXJpu057bL4M

Lorena del Encinar Cano Lucas, M. C. M. y A. V. B. F. (2018). Onicofagia. FMC. Formación Médica Continuada En Atención Primaria. https://www-clinicalkey-es.ezproxy.unex.es/\#!/content/playContent/1-s2.0S1134207217302086?returnurl=https:\%2F\%2Flinkinghub.elsevier.com\%2Fretrieve\%2Fpii\%2FS11342072 17302086\%3Fshowall\%3Dtrue\&referrer=https:\%2F\%2Fdialnet.unirioja.es\%2F

Moreno Vázquez, K., María, R., Olivera, P., Cano, A. S., Rosales, V. N., Antonio, J., Deseuza, S., Jurado, F., Cruz, S., \& Ubbelohde Hennigsen, T. (2007). Dermatología Rev Mex Volumen 51, Núm. 5, septiembre-octubre. www.revistasmedicasmexicanas.com.mx

Pelios, L., Morren, J., Tesch, D., \& Axelrod, S. (1999). THE IMPACT OF FUNCTIONAL ANALYSIS METHODOLOGY ON TREATMENT CHOICE FOR SELF-INJURIOUS AND AGGRESSIVE BEHAVIOR. Journal of Applied Behavior Analysis, 32(2), 185-195. https://doi.org/10.1901/jaba.1999.32-185 
Psacan, P., Reich, A., Grzesiak, M., \& Szepietowski, J. C. (2014). Onychophagia is associated with impairment of quality of life. Acta Dermato-Venereologica, 94(6), 703-706. https://doi.org/10.2340/00015555-1817

Salgado Serrano, G. N., \& Oropeza, R. (2012). Onicofagia: estudio de caso de una mujer adulta. Uaricha Revista de Psicología, 9(20), 71-82. https://www.mendeley.com/catalogue/b9973270-2abd-31b9-91adf33a56ca21a6/?utm_source=desktop\&utm_medium=1.19.8\&utm_campaign=open_catalog\&userDocument Id =\%7Bcbdf3825-aad1-34c1-923b-ed3717bfbf38\%7D 